\title{
The Role of Heat Shock Protein 70 kDa in Asthma
}

This article was published in the following Dove Press journal:

Journal of Asthma and Allergy

\author{
Marina Shevchenko (1D \\ Ekaterina Servuli $\mathbb{I D}^{1,2}$ \\ Zarema Albakova (D) ${ }^{1,3}$ \\ Leonid Kanevskiy' \\ Alexander Sapozhnikov ${ }^{1,3}$ \\ 'Department of Immunology, Shemyakin \\ and Ovchinnikov Institute of Bioorganic \\ Chemistry, Russian Academy of Sciences, \\ Moscow, Russia; ${ }^{2}$ Department of \\ Experimental Physiology, Institute of \\ Biomedical Problems, Russian Academy \\ of Sciences, Moscow, Russia; \\ ${ }^{3}$ Department of Biology, Lomonosov \\ Moscow State University, Moscow, Russia
}

Correspondence: Marina Shevchenko Department of Immunology, Shemyakin and Ovchinnikov Institute of Bioorganic Chemistry, Russian Academy of Sciences, Miklukho-Maklaya Street 16/I0, Moscow I I7997, Russia

Tel/Fax +749533040I I

Email mshevch@gmail.com

\begin{abstract}
Asthma is a complex chronic disorder of the airways, affecting immune and structural cells and inducing both protein and tissue remodeling. Heat shock proteins $70 \mathrm{kDa}$ (HSP70s) are highly conserved members of the stress-induced family, possessing precisely described chaperone activity. There is growing evidence of a tight relationship between inflammatory diseases of different origins and the elevation of local HSP70 expression and secretion. Although extracellular HSP70 does not serve as a common marker of asthma, elevated HSP70 levels have been detected in the peripheral blood serum and sputum of patients with asthma, as well as in the bronchoalveolar lavage fluid of mice with induced allergic airway inflammation. Possessing diverse immunomodulating properties, extracellular HSP70 can manifest different activities in airway inflammatory processes and asthma, acting either as a pro-inflammatory trigger, or an anti-inflammatory agent. This review will discuss the effects and possible mechanisms concerning HSP70 implication in airway inflammation regulation in asthma. We examine ATPase and chaperone activities of HSP70 as potential modulators of immune responses in asthma. Given the crucial role of a chronic inflammatory response in asthma, understanding the effects of HSP70 on immune and structural cells may reveal new perspectives for the therapeutic control of inflammation.
\end{abstract}

Keywords: heat shock protein $70 \mathrm{kDa}$, asthma, allergic airway inflammation, immune cells, ATP

\section{Introduction}

Asthma is a chronic inflammatory disease that includes several diverse phenotypes traditionally divided into "allergic" and "nonallergic" forms. ${ }^{1}$ Recently, based on molecular mechanisms, asthma phenotypes were combined into two basic endotypes: "T2-high" and "Non-T2". 2 The first is associated with the T helper 2 (Th2)-mediated response, and the other with a non-Th2-mediated response. ${ }^{2}$ T2-high and allergic asthma are also associated with eosinophilia and can be referred to as "eosinophilic," while non-allergic asthma is mostly associated with neutrophil-mediated inflammation. However, cases of non-allergic eosinophilic asthma have also been reported. ${ }^{1-3}$

Each endotype is characterized by different cytokine sets. Thus, allergic asthma is mediated by IL-4, IL-5, IL-9, and IL-13, while the dominant cytokines in nonallergic asthma are IL-1b, IL-8, IL-17, and IL-23. ${ }^{3,4}$ However, some features are common to both endotypes. The initiation of inflammation is driven by tissue cytokines (TSLP, IL-25, and IL-33), and both allergic and non-allergic asthma switch to chronic inflammatory processes, which manifest with structural cell damage, followed by airway remodeling. ${ }^{1-4}$ Besides the typical cytokines associated with asthma, some damage-associated molecular patterns (DAMP), including the $70-\mathrm{kDa}$ heat shock protein (HSP70), reportedly play an essential role in asthma. $^{5}$

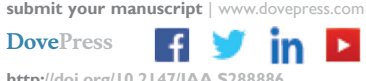

htp://doi.org/10.2147/JAA.s288886
Journal of Asthma and Allergy 2020:13 757-772 
The HSP70 is a family of chaperone proteins, that consists of 13 members in humans: HspA1A, HspA1B, HspA1L, HspA2, HspA5, HspA6, HspA7, HspA8, HspA9, HspA12A, HspA12B, HspA13, HspA14. ${ }^{6}$ Eight of them are expressed constitutively, but HspA1A, HspA1B, HspA5, HspA6, and HspA14 are stressinducible. ${ }^{6}$ Each HSP70 member has a definite intracellular location, and a number of these proteins were also found in the extracellular compartment, mostly in exosomes. ${ }^{6}$ All HSP70 members had some alternative names that were used before the unique nomenclature adoption. ${ }^{7}$ Hereafter the mentioned above HSP70 family members of humans and other species are referred to HSP70 if otherwise not purposely indicated by the authors.

Even though circulating HSP70 does not serve as a specific marker of asthma, it has been observed at high levels in the sputum and serum of patients with asthma, as well as in the bronchoalveolar lavage (BAL) fluid of mice in a model of induced allergic airway inflammation. ${ }^{8,9}$ Several reports demonstrate both the pro-inflammatory and anti-inflammatory activity of HSP70 during the course of inflammation. ${ }^{10-14}$ However, a lot is yet to be understood. This review will discuss the effects of HSP70 in the context of asthma and airway inflammation.

\section{HSP70 as a Potential Trigger of Asthma}

Several members of the HSP70 family detected in other species are reported as allergens (Table 1). This relation to allergens was determined, based on the presence of antibodies to these proteins in the peripheral blood sera of patients with allergies. Most HSP70 allergens are listed as inhaled allergens, except Mala s 10 from Malassezia sympodialis yeast, which colonizes the skin, and Aed a 8 from the Aedes aegypti mosquito. Antibodies to Aed a 8 have been identified in allergic individuals with asthma and/or rhinitis, and those sensitized to mosquitoes. ${ }^{15}$ Despite the high homology among proteins of the HSP70 family, none is reported as an auto-allergen. ${ }^{16}$ Recently, HSP70 from Chinese birch pollen extract was found to be the dominant protein that induced a strong specific IgE-response in a mouse model of birch pollen airway inflammation, suggesting it may play a role in Chinese birch pollen allergy. ${ }^{17}$

The antibodies that recognize recombinant human HSP70 have been found in the plasma of patients with asthma. Moreover, correlations were observed among asthma severity, increased IgE and IL-4 levels, and IgG to self (human) HSP70. ${ }^{18}$ Based on these findings, Hosseini and coauthors ${ }^{19}$ performed a clinical trial, in which they claimed that anti-HSP70 is a novel risk factor for asthma. They showed that anti-Hsp70 antibodies (IgG, IgA, and IgM) were elevated in the peripheral blood of placebotreated patients with asthma. Signs of HSP70 involvement in allergic diseases have also been identified in patients with allergic dermatitis. In particular, serum Mala s 10-specific IgE, derived from patients with allergic eczema or allergic dermatitis, showed high levels of cross-reactivity to self HSP70. ${ }^{20}$

Thus, in accordance with the information above, upon inhalation, some exogenous proteins from the HSP70 family can promote allergic airway inflammation in sensitive people. Moreover, the data about the cross-reactivity of anti-HSP70 antibodies to self HSP70 in atopic patients $^{20}$ give evidence that under certain conditions, exogenous HSP70 can trigger reactivity to self HSP70. One possible reason for this is the highly conserved structural sequences of exogenous allergic proteins of the HSP70 family and self HSP70 proteins.

Table I HSP70 Allergens of Different Species

\begin{tabular}{|c|c|c|}
\hline HSP70 Allergens & Species & References \\
\hline Alt a 3 & Alternaria alternate (mold) & Breitenbach \& Simon-Nobbe $2002^{1 / 3}$ \\
\hline Pen c 19 & Penicillium citrinum (mold) & Shen et al $1997^{114}$ \\
\hline Mala $s 10$ & Malassezia sympodialis (skin colonizing yeast) & Andersson et al $2004^{20}$ \\
\hline Der $f 28$ & Dermatophagoides farina (American house dust mite) & Aki et al $1994 ;^{115}$ An et al $2013^{116}$ \\
\hline Der $p 28$ & Dermatophagoides pteronyssinus (European house dust mite) & Liu et al $2018^{117}$ \\
\hline Tyr p 28 & Tyrophagus putrescentiae (mold mite) & Cui et al $2016^{118}$ \\
\hline Cor a 10 & Corylus avellana (European hazel) & Gruehn et al $2003^{119}$ \\
\hline Aed a 8 & Aedes aegypti (yellow fever mosquito) & Cantillo et al $2017^{15}$ \\
\hline Cla h Hsp70 & Cladosporium herbarum (mold) & Breitenbach \& Simon-Nobbe $2002 ;^{113}$ Zhang et al $1996^{120}$ \\
\hline Blo t Mag29 & Blomia tropicalis (dust mite) & Simon-Nobbe et al $2008^{121}$ \\
\hline
\end{tabular}

Note: In accordance with the database AllFam (http://www.meduniwien.ac.at/allfam/). ${ }^{16}$ 


\section{Elevation of HSP70 Expression and Secretion in Asthma}

More than 20 years ago, the increased expression of HSP70 in airway epithelial cells and alveolar macrophages of people with asthma was demonstrated by immunostaining in vitro. ${ }^{21}$ Further, the elevated expression of HSP70 mRNA was observed in peripheral blood mononuclear cells (PBMC) of patients with asthma during acute episodes. ${ }^{22}$ Later, the local and systemic elevation of extracellular HSP70 levels were shown to be correlated with the severity of inflammation in asthma. ${ }^{8}$ Furthermore, an association between increased plasma HSP70 levels and airway neutrophilia has been reported. ${ }^{8}$ In addition, a correlation between sputum HSP70 level and lymphocyte number has been detected in patients with asthma. ${ }^{8}$

Recently, Huang and coauthors ${ }^{5}$ compared the release of DAMP from structural airway cells and infiltrated immune cells in the sputum of patients with asthma, chronic obstructive pulmonary disease (COPD), and those with both asthma and COPD. Compared with the control group, the levels of HSP70 were elevated in the sputum of all three experimental groups (asthma group, COPD group, as well as the asthma-COPD overlap syndrome group). Interestingly, multiparametric analysis indicated a correlation between the percentage of neutrophils and the concentrations of HSP70. ${ }^{5}$ Elevated levels of extracellular HSP70 in the airways have also been reported in other studies using animal models of allergic airway inflammation. Thus, HSP70-rich exosomes have been identified in the BAL fluid of mice that received the olive pollen allergen $24 \mathrm{~h}$ after intranasal allergen application. ${ }^{23}$ Interestingly, in this study, to detect HSP70 in exosomes authors used antibodies to Hsc70 (HspA8 according to the unique nomenclature) ${ }^{7}$ that is known to be constitutive, but not stress inducible. ${ }^{23}$ Elevated BAL HSP70 production has also been observed in an ovalbumin-induced asthma model in mice. The kinetics of extracellular HSP70 levels indicated significant elevations at 24 and $48 \mathrm{~h}$ after the allergen challenge, compared to control mice. ${ }^{9}$

In summary, the elevated expression (of both the mRNA and protein) and secretion of HSP70 are associated with asthma, and are mostly detected in the acute phase of inflammation or exacerbation.

\section{Effects of HSP70 in Airway Inflammation}

Both pro-inflammatory and anti-inflammatory effects have been reported for HSP70 in airway inflammation. ${ }^{10,14}$ The effects of HSP70 can vary based on the origin (self or exogenous) of the protein and the microenvironment, such as receptors that can bind HSP70, and cells that express those receptors. The study design, such as specific methods of HSP70 expression stimulation or the pathological conditions during HSP70 application, can also influence the effects of HSP70. Moreover, some conditions can affect both inflammation and HSP70 expression independently. ${ }^{9-14,24-29}$

Traditionally, it has been suggested that intracellular HSP70 are cytoprotective chaperones, which when secreted to the extracellular space, possess proinflammatory properties and serve as alarmins. ${ }^{24}$ Thus, besides the potential immunogenicity and allergenicity of exogenous HSP70, extracellular self HSP70 can also exhibit pro-inflammatory effects during airway inflammation, which has been demonstrated in vitro using both primary cell cultures and various cell lines. The application of HSP70 to primary human lung fibroblasts can induce IL8 production. ${ }^{25}$ Moreover, using small interfering RNA, researchers have demonstrated that HSP70 are implicated in the induction of IL-8 production, in response to other stimuli, such as cigarette smoke extract. ${ }^{25}$

The treatment of human bronchial epithelial cells (16HBE14o-) with recombinant Hsp72 (HspA1A $)^{7}$ induces a dose-dependent increase in $I L-8$ gene expression; and pretreatment of cells with polymyxin B to prevent the side effects of endotoxins that contaminate recombinant Hsp72 does not alter the effects of Hsp72. ${ }^{26}$ Wheeler and coauthors ${ }^{27}$ showed that recombinant Hsp72 treatment leads to increased IL- 8 and TNF- $\alpha$ production by both primary human neutrophils and differentiated (by the addition of DMSO) HL-60 cells. The authors also pretreated cells with polymyxin B to prevent the effects of endotoxins. Similarly, elevated expression of TNF- $\alpha$ and $\mathrm{KC}-$ the murine functional homolog of human IL8 - has been observed in murine bone marrow neutrophils treated with human recombinant Hsp72. ${ }^{27}$ Using THP-1 cells differentiated by phorbol-myristate-acetate (PMA) activation into macrophage-like cells, Hulina and coauthors $^{28}$ showed dose-dependent and time-dependent production of IL-1a and IL-8, in response to treatment with recombinant human HSP70 (both HspA1A and HspA1B) ${ }^{7}$ containing low levels of endotoxin contamination. Later, the same group demonstrated the production of IL- 6 and IL- 8 by the human lung epithelial cell line NCIH292, after incubation with extracellular recombinant HSP70. $^{29}$ 
Some in vivo experiments have demonstrated the proinflammatory activity of HSP70. In one study, BALB/c mice were challenged with a single intratracheal inhalation of recombinant human HSP72, and euthanized 4 h later. ${ }^{27}$ The Hsp72 inhalation significantly elevated the production of TNF- $\alpha$ and KC. This in turn, attracted neutrophils to the airways and subsequently, increased levels of myeloperoxidase were detected in the murine BAL fluid, $4 \mathrm{~h}$ after Hsp72 application. ${ }^{27}$ However, these in vivo experiments did not permit any distinguishing between the effects of Hsp72 and those of endotoxin contaminants. As is well known from the standard ovalbumin-induced asthma mouse model, endotoxin contamination can affect the immune response to antigens. Thus, lipopolysaccharide (LPS)-free ovalbumin administration can induce IL-4, IL5, and IL-13 secretion and eosinophil infiltration, while LPS-rich ovalbumin application can also increase IL-1b, TNF- $\alpha$, and KC, as well as the infiltration of both eosinophils and neutrophils. ${ }^{30}$

To clarify the role of Hsp70 in airway inflammation, Yombo and coauthors ${ }^{31}$ recently demonstrated that HSP70 double knockout (Hsp70.1/.3-/-) mice that lack inducible HSP70 genes show significantly reduced Th2-mediated airway inflammation. Using the model of allergic airway inflammation induced by Schistosoma mansoni soluble egg antigen (SEA), those authors demonstrated that in the absence of inducible HSP70 gene expression, the total BAL cell number and eosinophil level, as well as IL-4, IL-5, and IL-13 cytokine levels, are significantly reduced. Simultaneously, the levels of neutrophils and lymphocytes in the BAL fluid of HSP70.1/.3-/- mice are significantly elevated in response to SEA administration, compared to those in the BAL fluid of wild type mice that received SEA. ${ }^{31}$ Notably, sensitization and challenge with SEA did not modify HSP70 gene expression in wildtype mice. Using bone marrow chimera and in vitro experiments, those authors showed that the loss of HSP70 is critical for $\mathrm{Th} 2$ response, and the absence of $\mathrm{T}$ cell activation leads to the attenuated airway inflammation observed in HSP70.1/.3-/- mice. ${ }^{31}$

Using the same mouse strain (HSP70.1/.3-/-) and a model of induced respiratory distress syndrome, a significant increase in lung injury and elevated levels of IL- 6 and TNF- $\alpha$ were observed in the absence of inducible HSP70. ${ }^{32}$ Thus, besides its pro-inflammatory effects, HSP70 can also display anti-inflammatory activity in the respiratory tract during inflammatory processes. Such activity has also been demonstrated in the murine model of bleomycin-induced lung fibrosis, where the induction of increased levels of HSP70 inhibits the progression of inflammation and fibrosis. ${ }^{33,34}$

Prado and coauthors ${ }^{23}$ detected increased levels of the constitutive form of $\mathrm{Hsc} 70$ (HspA8) ${ }^{7}$ in BALF-derived exosome that were yielded from mice with induced tolerance to the allergen. Moreover, preventive intranasal immunization of mice with HSP70-rich exosomes blocked the development of olive pollen allergen (Ole e 1)-induced allergic airway inflammation, and particularly, significantly reduced IL-5, eosinophilia, and allergen-specific IgE. ${ }^{23}$ Using a mouse model of ovalbumin-induced allergic airway inflammation, the direct protective effect of oropharyngeal HSP70 application in the acute phase of inflammation has been demonstrated. ${ }^{9}$ Particularly, the application of murine HSP70 prevents airway eosinophilia and suppresses the allergen-induced elevation of both the total BAL cell number and the levels of pro-allergic cytokines, such as IL-4, IL-5, and IL-13. ${ }^{9}$ Notably, in this study, airway inflammation was induced with ovalbumin grade 5, which is contaminated with endotoxin, and therefore provoked mixed eosinophilic and neutrophilic inflammation. Interestingly, the administration of HSP70 during the acute phase of inflammation in mice induced the retention of neutrophils in the BAL fluid, compared with mice affected by induced airway inflammation that did not receive HSP70. ${ }^{9}$ In vitro, HSP1A1 prevented neutrophil necrosis or NETosis and facilitated a proapoptotic neutrophil state. ${ }^{9}$

Thus, as it is shown above, several in vivo and in vitro models demonstrate both pro-inflammatory and antiinflammatory effects of HSP70 in the airways. In the case of pro-inflammatory effects, the following events are suggested: HSP70 stimulates the production of IL-1 alpha, IL-6, and IL-8 by the epithelial cells and alveolar macrophages, which attract neutrophils. The neutrophils in turn produce TNF- $\alpha$ and IL- 8 (or KC in mice), which act as autocrine stimulators of the neutrophil-mediated response. Interestingly, in the case of anti-inflammatory activity, HSP70 also affects neutrophils, by preventing their hyperactivation. Regarding allergic inflammation, inducible HSP70 (among which HspA1A, HspA1B, HspA6, HspA7 and HspA14) ${ }^{7}$ contributes to direct Th2 activation and the maintenance of a pro-allergic response, including eosinophilia, and elevated levels of IL-4, IL-5, and IL-13. However, exogenously supplied HSP70 downregulates eosinophilia, and levels of pro-allergic cytokines and allergen-specific IgE. To address the question about 
the dualistic effects of HSP70 in airway inflammation, further, we will focus on the molecular and cellular mechanisms of HSP70 action.

\section{HSP70 Binding Receptors and Their Role in Asthma}

One key point in establishing the concept of the immunomodulatory effects of HSP70 is defining the receptor responsible for the recognition of HSP70 by immune or structural cells during airway inflammation. As HSP70 can be involved in interactions with a receptor in a membranebound or an extracellular form, in complex with a peptide and in ATP- and ADP-binding states, definition of the unique receptor is quite problematic. Calderwood et $\mathrm{al}^{35}$ proposed that HSP70 mediates its activity by interacting with several cell surface receptors that can bind HSP70 or the HSP70-peptide complex. To date, these HSP70 binding receptors are represented as CD91, several scavenger receptors that belong to the family of $\mathrm{C}$-type lectin receptors (such as LOX-1 and SCARF1), C-type lectin-like receptor NKG2D, as well as the sialic acid-binding immunoglobulin-type lectins, Siglec-5 and Siglec-14. ${ }^{35-38}$ Furthermore, a heterodimeric cluster of TLR2/TLR4 and CD14, with and without the contribution of SCARF1, is reportedly involved in the binding of HSP70. ${ }^{39,40}$ A recent study also provided the basis for the interaction between HSP70 and the receptor of advanced glycation end products (RAGE). ${ }^{41}$

With the exception of RAGE, little is known about the role of the above-mentioned receptors in airway inflammation and asthma. At the same time, RAGE is reportedly the "major mediator of pulmonary inflammatory responses." It is constitutively highly expressed in the lungs of healthy adults, on the airway epithelial, endothelial, and smooth muscle cells, as well as on immune cells, including macrophages, dendritic cells (DCs), eosinophils, $\mathrm{T}$ cells, and B cells. ${ }^{42}$ The levels of endogenous and soluble RAGE are increased in the sputum samples of patients with asthma. $^{42}$ Furthermore, RAGE KO mice produce normal amounts of IL-4, in response to the house dust mite (HDM) but show attenuated IL-5 and IL-13 responses. In addition, RAGE promotes the expression of IL-33 and subsequent accumulation of ILC2s in the lungs of mice at the onset of allergic inflammation. ${ }^{42}$ Based on the facts above, we can conclude that although RAGE can mediate the pulmonary allergic inflammatory response, it is not known, whether HSP70-RAGE interaction triggers this response. The pro-inflammatory activity of RAGE is evidently driven by binding with the RAGE ligand, HMGB1, the expression of which is also elevated in asthma. ${ }^{42,43}$ Interestingly, the expression of HMGB1 in asthma is negatively regulated by HSF1, the transcriptional factor responsible for the expression of HSP70. ${ }^{43}$ Thus, although RAGE expression is associated with asthma, there is no direct proof that binding of HSP70 to RAGE can exacerbate asthmatic inflammation. Conversely, there is evidence that HSP70 expression can restrict the pro-inflammatory activity of RAGE by downregulating of HMGB1. ${ }^{42,43}$

Expression of CD91 has been observed on some structural cells, such as endothelial and smooth muscle cells and a wide range of immune cells, including DCs, monocytes, macrophages, and $\mathrm{B}$ and $\mathrm{T}$ lymphocytes. ${ }^{44}$ Interestingly, CD91 has been identified as a negative regulator of DC-mediated Th2 immune responses during HDM-induced eosinophilic airway inflammation. ${ }^{45}$ Whether HSP70-CD91 interaction involves in such suppression of the allergic immune response is currently unknown, however, it can be a hypothesis for the further research of the mechanisms of immunomodulatory activity of HSP70.

Both SCARF1 and LOX-1 are expressed on activated endothelium and antigen-presenting cells (APCs). ${ }^{38,40}$ Recently, the role of C-type lectin receptors in asthma was highlighted. $^{46}$ Some HDM or fungal allergens are recognized by C-type lectin receptors, mostly dectin receptors and mannose receptors. However, no direct evidence exists of the implication of SCARF1 and LOX-1 in allergic airway inflammation and asthma. Nevertheless, the expression of LOX-1 on the endothelium affects (slow down) neutrophil turnover, which could be important in the inflammatory process in the airway. ${ }^{47}$ These facts suggest the HSP70 interaction with SCARF1 or LOX-1 on neutrophils can be responsible for the neutrophil retention, which was associated with the inflammation resolution in experiments describing the protective effects of HSP70 in the asthma mouse model. ${ }^{9}$ Moreover, both SCARF1- and LOX-1-mediated endocytosis of apoptotic cells is necessary step for the resolution of inflammation. ${ }^{38}$

Several Siglecs have been implicated in the pathogenesis of asthma, particularly Siglec-8 (Siglec-F in mice) in allergic asthma, and Siglec-9 in severe neutrophilic asthma. $^{48-50}$ Neutrophils also express Siglec-5 and Siglec-14, both of which reportedly bind HSP70. Siglec5 and Siglec-9 are thought to be inhibitory, while Siglec14 is an activator that induces the production of IL-8 and 
TNF- $\alpha$ upon interaction with sialic acid. ${ }^{48-50}$ In accordance with this concept, the regulation of airway inflammation through the interaction of HSP70 with Siglecs has been demonstrated. Particularly, HSP70 stimulation through Siglec-5 acts as an anti-inflammatory signal, while stimulation through Siglec-14 is proinflammatory. ${ }^{37}$ The facts indicated above support the hypothesis that the effect of HSP70 in airway inflammation depends on both the presence of the certain cell type (e.g. neutrophils or eosinophils) and the current level of the certain Siglec expression on these cells. Such a hypothesis gives the mechanistic explanation of the multidirectional effects of HSP70 in inflammatory processes.

The effects of TLR activation in asthma, particularly TLR2 and TLR4, are dependent on many factors. ${ }^{51}$ Thus, multiple studies of TLR4 activation in asthma mouse models have shown either protection against, or exacerbation of asthma after exposure to endotoxins (LPS). ${ }^{30,51}$ The effect is dependent on LPS concentration and the route of exposure. In general, the administration of low doses of LPS together with the allergen induces a mixed Th2 and Th17 response, and the infiltration of both eosinophils and neutrophils to the airways; while the administration of high doses of LPS with the allergen induces a strong Th1 response. ${ }^{51}$

Simultaneously, endotoxin-free HSP70 - TLR4 interaction remains a matter of debate. Thus, Hulina et $\mathrm{al}^{28}$ demonstrated that HSP70, contaminated with a very low dose of LPS, can modulate the effects of LPS and lipoteichoic acid (LTA) interactions with TLR4 and TLR2, respectively. However, other researchers have shown opposite responses to LPS-free HSP70. Thus, opposite to contaminated with LPS recombinant Plasmodium falciparum HSP70 obtained in E. coli XL1 Blue, LPS-free HSP70 obtained in E. coli ClearColi BL21 and in Brevibacillus choshinensis, did not stimulate production of IL-6 and IL-8 in murine bone marrow-derived DCs. ${ }^{52}$ Furthermore, the addition of LPS-free HSP70 to LPSactivated human peripheral blood monocytes downregulates TNF- $\alpha$ and IL-6 secretion. ${ }^{53}$ Notably, in the same study, the downregulation of TLR4 expression under LPSfree HSP70 exposure has not been demonstrated. Other researchers have identified HSP70 as an effector molecule downstream of TLR4 in the inflammatory signaling cascade, and have shown that $\mathrm{KC}$ production in response to TLR activation is significantly reduced in B6-Hspala

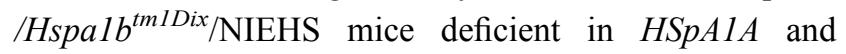

HSPA1B genes. ${ }^{54}$ Thus, the controversial effects of TLR4 activation in asthma and controversial data about HSP70TLR4 interactions require more detailed analysis. Additional studies are necessary to determine a single consistent hypothesis about the effects of HSP70mediated TLR4 activation in asthma.

The natural killer (NK) cell receptor, NKG2D, and NK cells are activated in asthma. ${ }^{55}$ NKG2D-deficient mice show no signs of allergic airway inflammation after exposure to the HDM allergen. ${ }^{56}$ However, several other studies showed that blocking of NKG2D by antibodies or genetic depletion have not significant influence on the development of asthma in different mouse models. ${ }^{55}$

The data shown above suggest, that most of the HSP70binding receptors have other ligands besides HSP70. Data showing that binding of HSP70 to its cognate or other immune receptors affects inflammation in asthma do not yet exist. The effects of activation of HSP70-binding receptors through interaction with HSP70 or other ligands in asthma depend on multiple factors such as the dose and nature of the ligand. The type of cell that expresses the HSP70-binding receptor is also very important in evaluating HSP70-mediated effects. Further studies are needed to explore whether HSP70 exerts its effects in asthma by binding to its cognate receptor or other immune receptors.

\section{Effects of HSP70 on Neutrophils}

The neutrophil-mediated response is mostly associated with non-allergic asthma and correlates with asthma severity and resistance to corticosteroid therapy. ${ }^{57}$ During the acute phase of allergic airway inflammation, as in any other inflammatory process, IL-8 secretion and subsequent neutrophil attraction is evident, as demonstrated by both clinical studies in human and experimental studies with mouse models of allergic airway inflammation. ${ }^{58,59}$ In cases of allergic asthma, neutrophils can suppress the progression of asthma. ${ }^{60}$

Besides the indirect neutrophil stimulation through alterations in the expression of neutrophil-attracting cytokines that were reported above, ${ }^{27}$ some direct effects of HSP70 on neutrophils have been demonstrated in experiments that were unrelated to airway inflammation. Thus, the dosedependent potential of stress-inducible Hsp72 (HspA1A) ${ }^{7}$ to stimulate chemotaxis of human neutrophils has been previously reported in in vitro models. ${ }^{61}$ Particularly, the participation of CD14 and TLR2 is reported to be associated with the mechanism of Hsp72-induced neutrophil chemotaxis. 
Another mechanism that can be implicated in HSP70mediated neutrophil recruitment from the bone marrow to the site of inflammation might be caused by the ability of HSP70 to interact with tetratricopeptide repeat (TPR) domain-containing protein co-chaperones. ${ }^{62}$ One such protein, HSP interacting protein (Hip), can also interact with CXCR2, which is expressed on mature neutrophils and is responsible for neutrophil recruitment from the bone marrow to peripheral blood. ${ }^{63}$ Moreover, the mutant Hip, which is unable to bind HSP70 (particularly, heat shock cognate protein $71 \mathrm{kDa}(\mathrm{Hsc} 70)$ or HspA8), ${ }^{7}$ induces attenuated chemotaxis of neutrophils. ${ }^{63}$ Thus, the elevation of extracellular HSP70 concentration at the site of inflammation may affect the chemotactic activity of neutrophils.

Neutrophils recruited to the site of inflammation can neutralize the pathogen through one of the following strategies: phagocytosis, reactive oxygen species (ROS) production, or neutrophil extracellular trap (NET) formation. Furthermore, ROS hyperproduction, as well as uncontrolled NETosis, are known to be important factors of asthma complications, in response to allergen provocation; while neutrophil apoptosis is advantageous for the resolution of inflammation. ${ }^{64-66}$ In vitro studies have demonstrated that HSP70 prevented bacterial antigen-induced ROS production and pro-inflammatory cytokine secretion by both human peripheral blood and mouse bone marrow neutrophils. ${ }^{67,68}$ Furthermore, HspA1A suppresses PMA-activated and AMLP-activated bone marrow neutrophil hyperactivation and prevents NETosis and necrosis. ${ }^{9}$ Simultaneously, HspA1A facilitates spontaneous apoptosis in the primary culture of bone marrow neutrophils. ${ }^{9}$ Recently, the contribution of ATP to the NETosis amplification was demonstrated. ${ }^{69}$ Thus, the inherent ATPase activity of HSP70 can be considered as one of the potential mechanisms of NETosis prevention (Figure 1).

In addition, the indirect effects of HSP70 regarding the neutrophil-mediated immune response are noteworthy. Some of these effects have been described above and are related to the production of neutrophil-attractant cytokines, such as IL-8 (or KC) and IL-1 $\beta$ by epithelial cells or macrophages. ${ }^{27,28}$ Furthermore, it was recently shown, that the Th2-associated cytokine, IL-4, interacts directly with IL-4R $\alpha$ on bone marrow neutrophils and blocks GMCSF-induced neutrophil recruitment to the site of inflammation. ${ }^{70}$ Moreover, neutrophil depletion can lead to a significant increase in Th2 cytokine

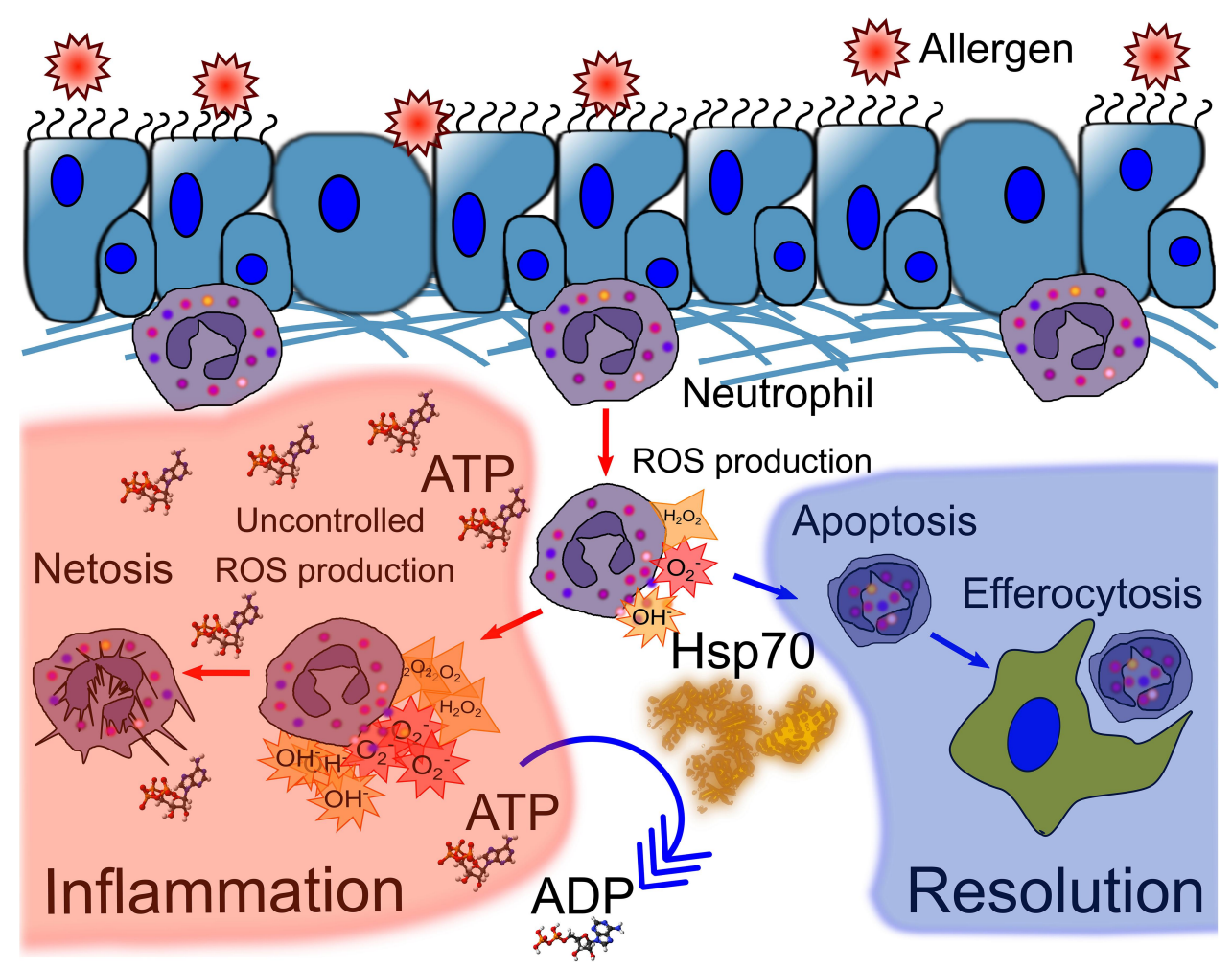

Figure I HSP70 prevents neutrophil hyperactivation at the site of inflammation. Production of reactive oxygen species (ROS) is one mechanism of neutrophil defense against pathogens; however, the trigger of ROS production can also cause damage to host tissues or damage-associated molecular patterns (DAMPS). ATP is known to stimulate ROS production. Uncontrolled ROS production leads to NETosis and inflammation progression. HSP70, as an ATPase, can dephosphorylate ATP to ADP, which is less pyrogenic than ATP, and thus prevent uncontrolled ROS production. Moreover, HSP70 was involved in switching from necrosis and NETosis of neutrophils to apoptosis. Apoptosis and efferocytosis of apoptotic neutrophils are associated with inflammation resolution. 
production by ILC2 during the early stages of allergic airway inflammation and amplifies the progression of inflammation. ${ }^{60}$ By reducing IL-4 levels in BAL fluid (as reported for the antiinflammatory effects of HSP70), ${ }^{9,71}$ HSP70 potentially prevents neutrophil retention in the bone marrow and facilitates neutrophil recruitment (Figure 2).

Thus, the indirect effects of HSP70 on the neutrophilmediated immune response can be the link between the proinflammatory and anti-inflammatory activity of HSP70 in allergic airway inflammation. By recruiting neutrophils, exogenous HSP70 suppresses the Th2-associated immune response in inflammation (Figure 2). At the same time, although elevated levels of extracellular HSP70 induce the recruitment of neutrophils, HSP70 prevents both neutrophil hyperactivation and the further progression of inflammation (Figure 1).

\section{HSP70-Mediated Effects on APCs, Antigen Presentation, and T Cell Activation: Implications in Allergic Asthma}

APCs play a pivotal role in asthma, particularly in allergic airway inflammation. Professional APCs include DCs, macrophages, and B cells. APCs recognize, internalize, and present peptides derived from allergenic proteins in complex with major histocompatibility complex (MHC) II, and in this way, activate adaptive immunity by inducing clonal allergen-specific Th2 expansion. ${ }^{72}$ In the atopic phenotype, Th2 cells are key cells of the adaptive immune system responsible for the hyperimmune reaction. ${ }^{73,74}$ Thus, the impact of HSP70 on APCs in allergic sensitization and allergic airway inflammation is of great interest.

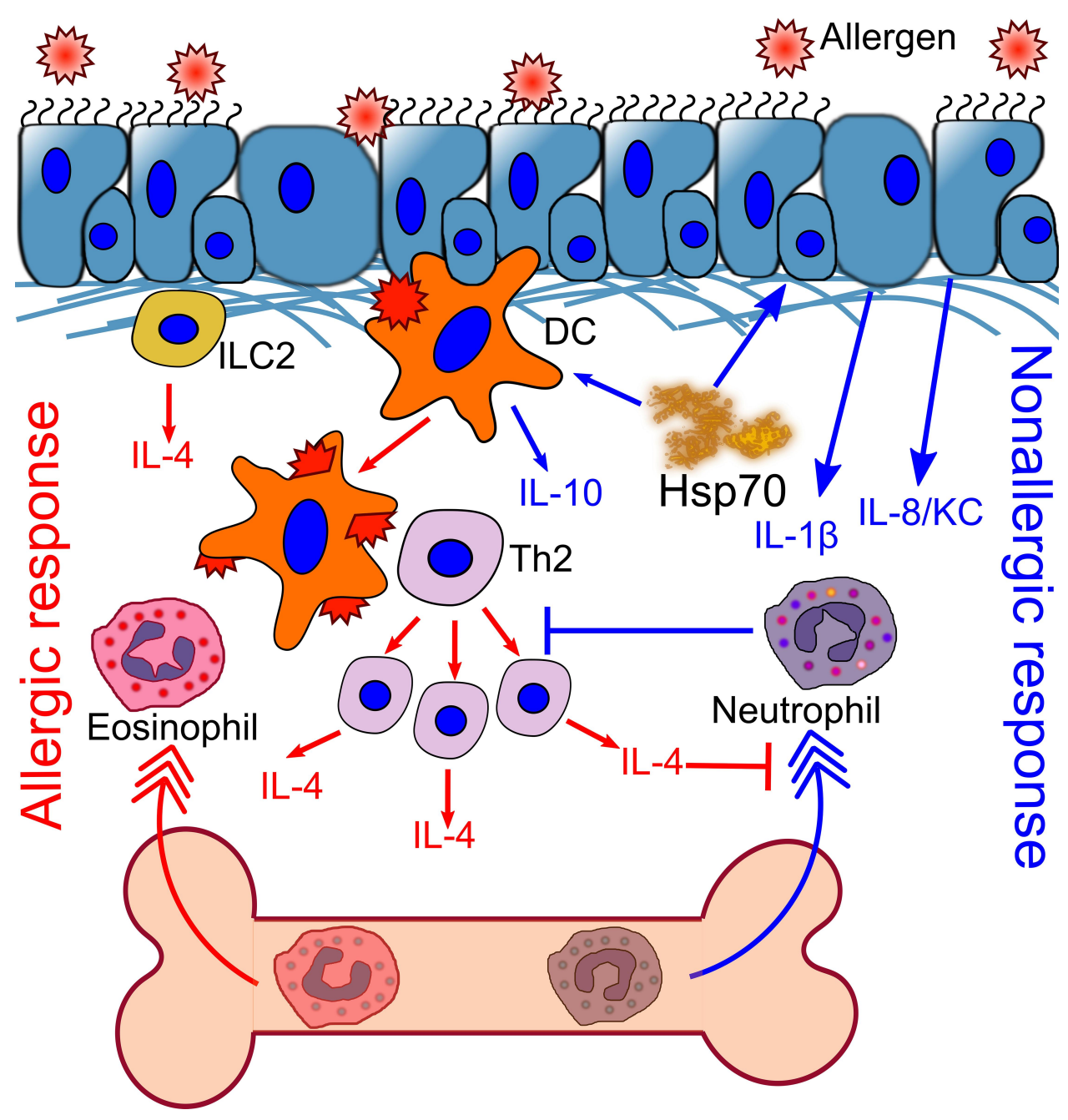

Figure 2 HSP70 induces neutrophil recruitment to the site of inflammation. Exogenous substance challenge induces neutrophil recruitment to the airways. However, inhaled allergen promotes IL-4 production by ILC2, and further by DC-activated Th2 cells. IL-4 concentration is increased locally and systemically. IL-4 blocks neutrophil recruitment, which promotes the recruitment of eosinophils and stimulates allergic airway inflammation. HSP70 activates the production of IL-I $\beta$ and IL-8 by epithelial cells, which attract neutrophils to the periphery. Neutrophils block Th2 proliferation and allergic airway inflammation. HSP70 also prevents DC maturation and induces IL-10 production. 
In the early studies that examined the potential of HSP70 to stimulate antitumor immunity, it has been shown that extracellular HSP70 has significant stimulatory effects on APCs and subsequently facilitates the adaptive immune response. Particularly, HSP70 induces APC maturation and the secretion of pro-inflammatory cytokines such as TNF- $\alpha$, IL-1b, IL-12, IL-6, and GMCSF. $^{14,75}$ These effects were served as a mechanistic explanation of antitumor effect of exogenous HSP70. ${ }^{76,77}$ However, later, the results were not confirmed; opposite, the suppressive effect of HSP70 and the induction of the tolerogenic APC phenotype were demonstrated. ${ }^{13,78}$

The HSP70-mediated suppressive effects have been demonstrated by findings that demonstrate endotoxin-free recombinant mycobacterial, and human HSP70 prevents bone marrow-derived DC maturation and induces the tolerogenic phenotype in mature DCs. ${ }^{13,78}$ In the presence of endotoxin-free HSP70, immature monocyte-derived DCs show reduced capacity to prime $\mathrm{T}$ cells, and activate $\mathrm{T}$ cell proliferation. The proliferation rates of both total pools of activated $\mathrm{CD}^{+} \mathrm{CD} 25^{+} \quad \mathrm{T}$ cells and non-activated $\mathrm{CD} 3^{+} \mathrm{CD} 25^{-}$cells, including $\mathrm{T}$ helper $\mathrm{CD} 4^{+} \mathrm{CD} 25^{-}$and $\mathrm{T}$ cytotoxic $\mathrm{CD} 8^{+} \mathrm{CD} 25^{-}$cells, are significantly reduced in the presence of recombinant human endotoxin-free HSP70. Moreover, HSP70 inhibits the proliferation of $\mathrm{T}$ cells that have been pre-activated with IL-12 or phytohemagglutinin. ${ }^{13}$

Molecular mechanisms underlying the suppressive effects of HSP70 on the antigen-presenting activity of innate immune cells are still not completely understood. On one hand, HSP70-MHCII interaction does not affect the primary structure of the antigenic peptide, and therefore, cannot modulate specific $\mathrm{T}$ cell responses even in conditions of enhanced or suppressed antigen presentation. ${ }^{79}$ On the other hand, the observed effects can be caused by the antigenic properties of HSP70, as exogenous or endogenous HSP70 protein can undergo internalization, following the processing and presentation of different HSP70-derived peptides in the context of MHCII. Peptide fragments of HSP70 have been shown to be expressed in complexes with both MHCII and MHCI. ${ }^{80}$

Notably, peptides of both extracellular and intracellular HSP70 pools can accumulate on MHCII, either as a result of cross-presentation or autophagy. ${ }^{10,80}$ Thus, even when HSP70 enhances antigen presentation, the presentation of peptides from the extracellular HSP70 pool is simultaneously increased. As a result, the specificity of Th2 cells shifts. Alteration of Th2 response specificity is associated with the suppression of allergic inflammation during allergen-specific immune therapy. ${ }^{81,82}$ Altogether, the data above suggest the following: antigen-presentation of HSP70 peptides might modulate allergic airway inflammation by preventing a part of the intracellular pool of MHCII from participation in allergen processing and presentation by occupying the MHCII complex. In this way, HSP70 reduces the number of allergen-specific Th2 clones, which, in turn, results in a reduced level of allergen-specific IgE. Thus, the immune-modulatory activity of HSP70 might be exhibited by switching the allergic response to tolerance.

Moreover, HSP70 can inhibit the proliferation of $T$ lymphocytes both indirectly, by downregulating the stimulatory activity of APCs, and by direct influence on previously activated $\mathrm{T}$ cells. ${ }^{10,83} \mathrm{In}$ addition to the suppressive effects on APCs and T cell proliferation, recent studies on the implications of HSP70 in the development of autoimmune disorders have demonstrated the potential of HSP70 to elevate the number of regulatory T cells ( $\mathrm{T}$ regs). Injections of HSP70 upregulate total Foxp3 expression and increase the percentage of $\mathrm{CD}^{+} \mathrm{CD} 25^{+} \mathrm{Foxp}^{+}$ cells in lymph nodes. ${ }^{83,84}$

Taken together, we can assume that under specific conditions, HSP70 promotes the differentiation of naïve $\mathrm{T}$ cells to $\mathrm{CD} 4^{+} \mathrm{CD} 25^{+} \mathrm{Foxp}^{+}{ }^{+}$inducible regulatory $\mathrm{T}$ cells and prevents the differentiation of naïve $\mathrm{T}$ cells to the Th2 phenotype. ${ }^{10,85}$ Thus, HSP70 can suppress the activation and proliferation of $\mathrm{T}$ cells and inhibit the differentiation of naïve T cells to the Th2 phenotype, which makes it one of the key features of allergic airway inflammation.

\section{Chaperone Activity of HSP70 in Asthma}

While extracellular HSP70 reveal both pro-inflammatory and anti-inflammatory activity, intracellular HSP70 are traditionally considered to be cytoprotective chaperones. ${ }^{24}$ Functional chaperone activity of HSP70 is due to their ability to interact with the hydrophobic regions of denaturing (unfolded or misfolded) proteins. ${ }^{86}$ The substrate-binding domain of HSP70 that can recognize denaturing proteins is located at the C-terminus of the molecule. This domain is represented by the $15 \mathrm{kDa} \beta$ sheet region, that contains the hydrophobic groove. ${ }^{62,87}$ Interacting with hydrophobic regions of newly synthesized or damaged denatured proteins, HSP70s participate in the 
stress-induced cellular responses, that can lead to the reparation of cellular homeostasis or cell death. ${ }^{88}$

As HSP70 are localized in the different cell compartments including the nucleus, mitochondria, cytosol, ${ }^{6}$ they also administrate the translocation of the polypeptide chains into different cell organelles, including the endoplasmic reticulum (ER) ${ }^{88}$ In the ER, HSP70 (particularly, HspA5) ${ }^{7}$ are involved in the mechanisms of the ER stress and the unfolded protein response (UPR) that reportedly play a sufficient role in asthma and airway inflammation. ${ }^{89,90}$ The ER stress was shown to contribute to such asthma features as airway remodeling and mucus hyperproduction. ${ }^{91}$ Moreover, the overexpression of UPR sensors (that are localized in the ER membrane - inositol requiring enzyme (IRE) 1, activating transcription factor (ATF) 6, and PKR-like ER kinase (PERK)) was demonstrated in both ovalbumin-induced and HDM-induced animal asthma models as well as in the tissue samples of asthma patients. ${ }^{91,92}$ In the absence of the ER stress, IRE1 $\alpha$, ATF6, and PERK bind HspA5. During the unfolded protein accumulation in ER, HspA5 binds the unfolded proteins and dissociates from the interaction with the UPR sensors; the loss of the interaction triggers the UPR activation. ${ }^{90-92}$

In the case when the UPR is not sufficient to repair the damage, the fragment of ER can be degraded via macroautophagy. ${ }^{93}$ HSP70 (particularly, Hsc70 or HspA8) plays an essential role in protein degradation through the ubiquitin-proteasome system (UPS) and in all three types of autophagy: chaperon-mediated, microautophagy and macroautophagy. ${ }^{88,94}$ Co-expression of HSP70 and the autophagy marker LC3 was detected in the airway cell culture A549 under the exposure of carbon nanoparticles and reported as an indicator of chaperonmediated autophagy. ${ }^{95}$ In certain conditions, carbon nanoparticles can trigger or exacerbate airway inflammation in asthma, and chaperon-mediated autophagy can be potentially involved in inflammation regulation. ${ }^{95,96}$

The role of autophagy in asthma is widely discussed and thought to be associated with airway remodeling and severe asthma. ${ }^{97,98}$ Single nucleotide polymorphisms in autophagy-related gene $\operatorname{Atg} 5$ are associated with reduced lung function and airway remodeling. ${ }^{93,96}$ Also, the expression of $\operatorname{Atg} 5$ product was higher in lung tissue samples of asthma patients compared with nonasthmatic samples. ${ }^{97}$ Furthermore, in the ovalbumin-induced mouse model of asthma, the treatment with Atg5 short hairpin RNA inhibited autophagy together with asthma features such as airway hyperreactivity and eosinophilia. ${ }^{98}$ Activation of autophagy is also involved in the NET formation (that implication in asthma was described above) and in neutrophilic airway inflammation..$^{65,96}$

Thus, the facts above indicate that intracellular HSP70 contribute to ER stress-induced UPR, UPS, and autophagy. All these processes are observed in asthma, particularly severe asthma. Taking into account elevated expression of HSP70 in airway epithelial and immune cells of people with asthma, ${ }^{21,22}$ we can assume that the protective chaperone activity of intracellular HSP70 during the severe inflammation in asthma plays an essential role in the degradation of the damaged proteins and the removal of destroyed organelles from the cells.

\section{Potential Suppressive Effects of HSP70 ATPase Activity in Systemic and Local Allergic Airway Inflammation}

One of the main functional characteristics of the HSP70 family is ATPase activity. ${ }^{86,99}$ The nucleotide-binding domain (45 kDa) belongs to the highly conserved region of the primary amino acid sequence of HSP70 proteins. It is in close proximity to the N-terminus of the molecule and typically contains the actin-like ATPase protein superfamily (sugar kinase/heat shock protein 70/actin superfamily) ATP-binding center. ${ }^{100}$ The ATP-binding center of HSP70 is mainly represented by proline, tyrosine, phenylalanine, arginine, and glutamine, which are positioned at the specific places relative to each other. ${ }^{101}$ For different members of the HSP70 family, the positions can be shifted by one or two amino acid residues; however, the shift does not affect domain conformation. ${ }^{101}$

Moreover, HSP70 can bind to ATP with nanomolar affinity. ${ }^{99,102}$ Consecutive interaction of HSP70 with ATP, followed by interaction with a peptide or denatured protein in the presence of co-chaperone and adapter proteins, induces the ATPase activity of HSP70, and hydrolysis of ATP to ADP. ${ }^{103}$ The difference in peptide binding of ATP-bound and ADP-bound HSP70 isoforms is the basis of chaperone activity. ${ }^{88}$ However, chaperone activity can also be manifested by HSP70 alone in the presence of co-chaperones. The most well-described co-chaperones are members of the J-protein family, which facilitate ATP dephosphorylation, and the nucleotide exchange factors (NEFs), which are responsible for the removal of ADP from the HSP70-nucleotide complex as well as the TPR 
domain-containing proteins. ${ }^{62}$ Both J-proteins and NEFs have binding sites in the region of the nucleotide-binding domain. $^{62,87}$

Recent studies demonstrate that ATP plays an important role in the activation of APCs. ${ }^{104}$ Release of ATP to the extracellular space, as a result of damage, stress, or cell death, has been implicated in the development of allergic airway inflammation. ${ }^{105}$ In particular, a sharp increase in ATP levels in the BAL fluid of persons with asthma and in mouse models has been detected shortly after allergen provocation. ${ }^{106}$ Extracellular ATP binding to the $\mathrm{P} 2 \mathrm{X} 7$ receptor can activate different types of cells and increase the production of pro-inflammatory cytokines such as: IL-1, IL-6, IL-18, and TNF- $\alpha$ by macrophages; IL-1 $\beta$, IL-18, TNF- $\alpha$, and IL-23 by DCs; IL-8 and eosinophil cationic protein (ECP) by eosinophils; as well as histamine secretion by mast cells. Taken together, extracellular ATP upregulation can facilitate Th2-activation and hyperimmune reactions. ${ }^{107}$ Direct neutralization of ATP through the administration of the ATP-hydrolyzing enzyme apyrase can significantly reduce inflammatory cell infiltration and conducting airway obstruction in induced allergic airway inflammation. ${ }^{106}$

Under non-inflamed steady-state physiological conditions, exoferments ectonucleoside triphosphate diphosphohydrolases (E-NTPD)s regulate extracellular ATP levels. ${ }^{108}$ Both E-NTPD and HSP70 belong to the sugar kinase/heat shock protein 70/actin superfamily and possess similar conformations of the ATP-binding domain, which is sensitive to $\mathrm{Ca}^{2+}$ or $\mathrm{Mg}^{2+} .{ }^{109}$ The E-NTPDs manifest ATPase activity; however, owing to their membrane-bound location, they can only act on the cell surface, but not within the extracellular matrix, unlike HSP70. ${ }^{108,110}$ The role of HSP70 in the regulation of extracellular ATP concentrations has not yet been determined in vivo. However, in vitro studies have shown a reduction in extracellular ATP levels in the presence of HSP70 in culture filtrates of bone marrow-derived DCs. ${ }^{111}$ Taken together, increased extracellular HSP70 concentration at the site of allergic airway inflammation and ATPase activity of the protein support the hypothesis that HSP70 can downregulate extracellular ATP levels and suppress P2X7 activation by reducing the activation stimulus.

Thus, the neutralization of extracellular ATP (the proinflammatory stimulus activating APCs to promote allergic airway inflammation) by HSP70 from the extracellular pool can be considered one of the molecular mechanisms associated with the anti-inflammatory potential of HSP70.

\section{Discussion}

In the presented review, we surveyed the published results related to effects and potential regulatory mechanisms of HSP70 in asthma.

The literature data demonstrated that under certain conditions, some exogenous HSP70 can trigger specific $\mathrm{IgE}$ production and even autologous allergic-like response to self HSP70. Regarding these findings, it would be of great interest to investigate the potential of some exogenous HSP70, for example, Pen c 19, to induce allergic airway inflammation in the murine model. As it was shown, murine HSP70 (yielded from the homogenate of mouse liver and kidney) was not potent to induce eosinophilia and pro-allergic cytokine production when was injected and inhaled to mice in accordance with the scheme similar to ovalbumininduced allergic airway inflammation. ${ }^{9}$ Mouse model of Pen c 19-induced airway inflammation would help to investigate the mechanisms of cross-reactivity and selfprotein tolerance. In fact, tolerance is of great importance as the elevated HSP70 mRNA expression and HSP70 protein secretion were found in asthma patient cell and tissue samples.

Despite the clear implication of HSP70 in asthma, there is no a unique concept about the role of HSP70 in asthma. Both pro-inflammatory and anti-inflammatory activities of HSP70 are reported. The ambiguous effects are detected in experiments with animal models of allergic airway inflammation and even with in vitro cell cultures. One explanation of such effects comes from the findings of Fong et $\mathrm{al}^{37}$ who demonstrated that HSP70 stimulation through Siglec-5 acts as an anti-inflammatory signal, while stimulation through Siglec-14 is pro-inflammatory. Thus, the current cell state, particularly the level of expression of certain receptor interacting with HSP70, can be a determinant in triggering the pro-inflammatory or antiinflammatory effects.

The other explanation of the dualistic effects can be related to the HSP70 protein characteristics. While the traditional concept suggests intracellular HSP70 to be cytoprotective chaperones, and extracellular HSP70 act as alarmins, both of these forms are elevated in asthma, and some studies reported the suppressive effects of extracellular HSP70. Due to cell and tissue damage during the inflammatory processes, it is quite difficult to discriminate 
the effects of physiologically released HSP70 (secreted as a component of exosomes) and effects of HSP70 that appeared in the extracellular space due to cell necrosis. Moreover, exosomal HSP70 can be represented with the different HSP70 family members at the different phases of allergic airway inflammation and in nonallergic response. Some experiments directed to precise characterization of the certain HSP70 function in the inflammatory processes in asthma could be of great importance. Such experiments should also take into account the state of the protein (ATPbound or ADP-bound), and therefore, not only LPS contamination, but also the concentration of ATP, and $\mathrm{Ca}^{2+}$, and $\mathrm{Mg}^{2+}$ in HSP70 preparations should be verified.

The estimation of ATP contamination in HSP70 preparations is of great importance in the studies of the ATPase activity of HSP70. Due to inherent ATPase activity, HSP70 can be involved in the neutralization of extracellular ATP, which is known to trigger the inflammatory response in both Th2- and T17-mediated asthma inflammation. Thus, the potential of the extracellular HSP70 to neutralize ATP and subsequently suppress the inflammation in asthma must be further in detail investigated. Additionally, the application of HSP70 inhibitors, particularly, ATP analogs and ATPase activity modulators, is very valuable in the investigation of the suppressive potential of ATPase activity in the inflammation in asthma. Such inhibitors can be applied in both animal models and in vitro experiments with asthma patient primary cell cultures and tissue samples. ${ }^{112}$

It can also be suggested, that some stimulatory HSP70 effects that are referred to as pro-inflammatory, in some situations, can prevent the inflammation. In particular, the observed in vitro pro-inflammatory HSP70 effects that are directed at structural (primarily epithelial) and innate immune cells, act through the stimulation of IL-1 alpha, IL-6, TNF- $\alpha$, and IL-8 production, in vivo can trigger the neutrophil infiltration. In the case of allergic airway inflammation, stimulation of neutrophil response can suppress eosinophilia and Th2 proliferation. Thus, the neutrophil-mediated response and activation of HSP70 can downregulate allergic airway inflammation (Figure 2). Regarding this suggestion, the estimation of a shift in cytokine production under the HSP70 exposure in the mouse models of allergic airway inflammation should not be restricted to common pro-allergic cytokines (IL-4, IL-5, IL-13), but neutrophil-attracting cytokine (IL-1b, IL8 or $\mathrm{KC}$, as well as GMCSF) production should also be estimated.

\section{Conclusion}

Many facts are confirming the involvement of HSP70 in the inflammatory processes in asthma, however, the mechanisms of this involvement require further study. In mouse models of airway inflammation, both intracellular and extracellular HSP70 exhibit the anti-inflammatory activity that characterizes the protein as a promising molecule for the development of new therapeutic approaches to managing asthma. However, some facts demonstrate a proinflammatory activity that HSP70 can display in different experimental models. In this review, we provided evidence that both pro-inflammatory and anti-inflammatory effects resulted from the unique mechanisms of HSP70 action. Further understanding of the molecular mechanisms of the HSP70-mediated regulatory functions in airway inflammation may reveal new therapeutic possibilities for the treatment of patients with asthma.

\section{Acknowledgments}

The work was supported by the RSF: the material regarding HSP70 project 19-75-10120, the material about asthma project 20-75-00111.

\section{Disclosure}

The authors report no conflicts of interest in this work.

\section{References}

1. Lambrecht BN, Hammad H. The immunology of asthma. Nat Immunol. 2015;16(1):45-56. doi:10.1038/ni.3049

2. Kuruvilla ME, Lee FE-H, Lee GB. Understanding asthma phenotypes, endotypes, and mechanisms of disease. Clin Rev Allergy Immunol. 2019;56(2):219-233. doi:10.1007/s12016-018-8712-1

3. Boonpiyathad T, Sozener ZC, Satitsuksanoa P, Akdis CA. Immunologic mechanisms in asthma. Semin Immunol. 2019;46:101333. doi:10.1016/j.smim.2019.101333

4. Lambrecht BN, Hammad H, Fahy JV. The cytokine of asthma. Immunity. 2019;50(4):975-991. doi:10.1016/j.immuni.2019.03.018

5. Huang XL, Tan XY, Liang Y, et al. Differential DAMP release was observed in the sputum of COPD, asthma and asthma-COPD overlap (ACO) patients. Sci Rep. 2019;9:19241. doi:10.1038/s41598-01955502-2

6. Radons J. The human HSP70 family of chaperones: where do we stand? Cell Stress Chaperones. 2016;21(3):379-404.

7. Kampinga HH, Hageman J, Vos MJ, et al. Guidelines for the nomenclature of the human heat shock proteins. Cell Stress Chaperones. 2009;14(1):105-111. doi:10.1007/s12192-008-0068-7

8. Hou C, Zhao H, Li W, et al. Erratum to: increased heat shock protein 70 levels in induced sputum and plasma correlate with severity of asthma patients. Cell Stress Chaperones. 2011;16(6):663-671. doi:10.1007/s12192-011-0278-2

9. Shevchenko MA, Troyanova NI, Servuli EA, Bolkhovitina EL, Fedorina AS, Sapozhnikov AM. Study of immunomodulatory effects of extracellular HSP70 in a mouse model of allergic airway inflammation. Biochemistry (Mosc). 2016;81(11):1384-1395. doi:10.1134/S0006297916110158 
10. Borges TJ, Wieten L, van Herwijnen MJ, et al. The anti-inflammatory mechanisms of Hsp70. Front Immunol. 2012;3:95. doi:10.3389/fimmu.2012.00095

11. De Maio A. Extracellular Hsp70: export and function. Curr Protein Pept Sci. 2014;15(3):225-231. doi:10.2174/ 1389203715666140331113057

12. Satoh M, Shimoda Y, Akatsu T, Ishikawa Y, Minami Y, Nakamura M. Elevated circulating levels of heat shock protein 70 are related to systemic inflammatory reaction through monocyte toll signal in patients with heart failure after acute myocardial infarction. Eur $J$ Heart Fail. 2006;8(8):810-815. doi:10.1016/j.ejheart.2006.03.004

13. Stocki P, Dickinson AM. The immunosuppressive activity of heat shock protein 70. Autoimmune Dis. 2012;2012:617213. doi:10.1155/2012/617213

14. Wallin RP, Lundqvist A, More SH, von Bonin A, Kiessling R, Ljunggren HG. Heat-shock proteins as activators of the innate immune system. Trends Immunol. 2002;23(3):130-135. doi:10.1016/S1471-4906(01)02168-8

15. Cantillo JF, Puerta L, Puchalska P, Lafosse-Marin S, Subiza JL, Fernandez-Caldas E. Allergenome characterization of the mosquito aedes aegypti. Allergy. 2017;72(10):1499-1509. doi:10.1111/all.13150

16. Radauer C, Bublin M, Wagner S, Mari A, Breiteneder H. Allergens are distributed into few protein families and possess a restricted number of biochemical functions. $J$ Allergy Clin Immunol. 2008;121(4):847-852.e7. doi:10.1016/j.jaci.2008.01.025

17. Xie ZJ, Yin J. Chinese birch pollen allergy and immunotherapy in Mice. Inflammation. 2019;42(3):961-972. doi:10.1007/s10753019-00957-8

18. Yang M, Wu T, Cheng L, Wang F, Wei Q, Tanguay RM. Plasma antibodies against heat shock protein 70 correlate with the incidence and severity of asthma in a Chinese population. Respir Res. 2005;6(1):18. doi:10.1186/1465-9921-6-18

19. Hosseini SA, Zilaee M, Shoushtari MH, Dehcheshmeh MG. An evaluation of the effect of saffron supplementation on the antibody titer to heat-shock protein (HSP) 70, hsCRP and spirometry test in patients with mild and moderate persistent allergic asthma: a triple-blind, randomized placebo-controlled trial. Respir Med. 2018;145:28-34. doi:10.1016/j.rmed.2018.10.016

20. Andersson A, Rasool O, Schmidt M, et al. Cloning, expression and characterization of two new IgE-binding proteins from the yeast Malassezia sympodialis with sequence similarities to heat shock proteins and manganese superoxide dismutase. Eur $J$ Biochem. 2004;271(10):1885-1894. doi:10.1111/j.14321033.2004.04098.x

21. Vignola AM, Chanez P, Polla BS, Vic P, Godard P, Bousquet J. Increased expression of heat-shock-protein-70 on airway cells in asthma and chronic-bronchitis. Am J Respir Cell Mol Biol. 1995;13(6):683-691. doi:10.1165/ajrcmb.13.6.7576706

22. Tong WC, Luo WC. Heat shock proteins mRNA expressions by peripheral blood mononuclear cells in asthma and chronic bronchitis. Chin Med J. 2000;113(2):175-177.

23. Prado N, Marazuela EG, Segura E, et al. Exosomes from bronchoalveolar fluid of tolerized mice prevent allergic reaction. $J$ Immunol. 2008;181(2):1519-1525. doi:10.4049/jimmunol.18 1.2.1519

24. Asea A, Kraeft SK, Kurt-Jones EA, et al. Hsp70 stimulates cytokine production via a CD14-dependent pathway: a "chaperokine". Cell Stress Chaperones. 2000;5(5):491.

25. Li CJ, Ning W, Matthay MA, Feghali-Bostwick CA, Choi AMK. MAPK pathway mediates EGR-1-HSP70-dependent cigarette smoke-induced chemokine production. Am J Physiol Lung Cell Mol Physiol. 2007;292(5):L1297-L1303. doi:10.1152/ ajplung.00194.2006
26. Chase MA, Wheeler DS, Lierl KM, Hughes VS, Wong HR, Page K. Hsp72 induces inflammation and regulates cytokine production in airway epithelium through a TLR4- and NF-kappa B-dependent mechanism. J Immunol. 2007;179(9):6318-6324. doi:10.4049/jimmunol.179.9.6318

27. Wheeler DS, Chase MA, Senft AP, Poynter SE, Wong HR, Page K. Extracellular Hsp72, an endogenous DAMP, is released by virally infected airway epithelial cells and activates neutrophils via Toll-like receptor (TLR)-4. Respir Res. 2009;10(1):31. doi:10.1186/1465-9921-10-31

28. Hulina A, Rajkovic MG, Despot DJ, et al. Extracellular Hsp70 induces inflammation and modulates LPS/LTA-stimulated inflammatory response in THP-1 cells. Cell Stress Chaperones. 2018;23 (3):373-384. doi:10.1007/s12192-017-0847-0

29. Hulina-Tomaskovic A, Rajkovic MG, Jelic D, et al. Proinflammatory effects of extracellular Hsp70 on NCI-H292 human bronchial epithelial cell line. Int J Exp Pathol. 2019;100 (5-6):320-329. doi:10.1111/iep.12335

30. Mac Sharry J, Shalaby KH, Marchica C, et al. Concomitant exposure to ovalbumin and endotoxin augments airway inflammation but not airway hyperresponsiveness in a murine model of asthma. PLoS One. 2014;9(6):e98648. doi:10.1371/journal. pone.0098648

31. Yombo D, Mentik-Kane MM, Wilson MS, Wynn TA, Madala SK. Hsp70 is a positive regulator of airway inflammation and mucus hypersecretion through the increase of type 2 cytokine production by activated $\mathrm{T}$ cells in allergic asthma. Am J Respir Crit Care Med. 2019;199:A1053.

32. Singleton KD, Wischmeyer PE. Effects of HSP70.1/3 gene knockout on acute respiratory distress syndrome and the inflammatory response following sepsis. Am J Physiol Lung Cell Mol Physiol. 2006;290(5):L956-L961. doi:10.1152/ajplung.00 466.2005

33. Fujibayashi T, Hashimoto N, Jijiwa M, Hasegawa Y, Kojima T, Ishiguro N. Protective effect of geranylgeranylacetone, an inducer of heat shock protein 70, against drug-induced lung injury/fibrosis in an animal model. BMC Pulm Med. 2009;9:45. doi:10.1186/ 1471-2466-9-45

34. Tanaka K, Tanaka Y, Namba T, Azuma A, Mizushima T. Heat shock protein 70 protects against bleomycin-induced pulmonary fibrosis in mice. Biochem Pharmacol. 2010;80(6):920-931. doi:10.1016/j.bcp.2010.05.025

35. Calderwood SK, Theriault J, Gray PJ, Gong J. Cell surface receptors for molecular chaperones. Methods. 2007;43 (3):199-206. doi:10.1016/j.ymeth.2007.06.008

36. Basu S, Binder RJ, Ramalingam T, Srivastava PK. CD91 is a common receptor for heat shock proteins gp96, hsp90, hsp70, and calreticulin. Immunity. 2001;14(3):303-313. doi:10.1016/ S1074-7613(01)00111-X

37. Fong JJ, Sreedhara K, Deng LW, et al. Immunomodulatory activity of extracellular Hsp70 mediated via paired receptors siglec-5 and siglec-14. EMBO J. 2015;34(22):2775-2788. doi:10.15252/ embj.201591407

38. Murshid A, Borges TJ, Bonorino C, Lang BJ, Calderwood SK Immunological outcomes mediated upon binding of heat shock proteins to scavenger receptors SCARF1 and LOX-1, and endocytosis by mononuclear phagocytes. Front Immunol. 2020;10:3035. doi:10.3389/fimmu.2019.03035

39. Asea A, Rehli M, Kabingu E, et al. Novel signal transduction pathway utilized by extracellular HSP70 - role of toll-like receptor (TLR) 2 AND TLR4. J Biol Chem. 2002;277 (17):15028-15034. doi:10.1074/jbc.M200497200

40. Murshid A, Borges TJ, Lang BJ, Calderwood SK. The scavenger receptor SREC-I cooperates with toll-like receptors to trigger inflammatory innate immune responses. Front Immunol. 2016;7:226. doi:10.3389/fimmu.2016.00226 
41. Grunwald MS, Ligabue-Braun R, Souza CS, et al. Putative model for heat shock protein 70 complexation with receptor of advanced glycation end products through fluorescence proximity assays and normal mode analyses. Cell Stress Chaperones. 2017;22 (1):99-111. doi:10.1007/s12192-016-0746-9

42. Oczypok EA, Perkins TN, Oury TD. All the "RAGE" in lung disease: the receptor for advanced glycation endproducts (RAGE) is a major mediator of pulmonary inflammatory responses. Paediatr Respir Rev. 2017;23:40-49. doi:10.1016/j. prrv.2017.03.012

43. Shang LQ, Wang L, Shi XL, et al. HMGB1 was negatively regulated by HSF1 and mediated the TLR4/MyD88/NF-kappa B signal pathway in asthma. Life Sci. 2020;241:117120. doi:10.1016/j.lfs.2019.117120

44. Cappelletti M, Presicce P, Calcaterra F, Mavilio D, Della Bella S. Bright expression of CD91 identifies highly activated human dendritic cells that can be expanded by defensins. Immunology. 2015;144(4):661-667. doi:10.1111/imm.12418

45. Mishra A, Yao XL, Saxena A, et al. Low-density lipoprotein receptor-related protein 1 attenuates house dust mite-induced eosinophilic airway inflammation by suppressing dendritic cell-mediated adaptive immune responses. $J$ Allergy Clin Immunol. 2018;142(4):1066-1079.e6. doi:10.1016/j.jaci.201 7.10 .044

46. Hadebe S, Brombacher F, Brown GD. C-type lectin receptors in asthma. Front Immunol. 2018;9:733. doi:10.3389/fimmu.2018.0 0733

47. Patten DA, Shetty S. More than just a removal service: scavenger receptors in leukocyte trafficking. Front Immunol. 2018;9:2904.

48. Chen Z, Bai FF, Han L, et al. Targeting neutrophils in severe asthma via siglec-9. Int Arch Allergy Immunol. 2018;175(1-2):5-15. doi:10.1159/000484873

49. Macauley MS, Crocker PR, Paulson JC. Siglec-mediated regulation of immune cell function in disease. Nat Rev Immunol. 2014;14(10):653-666. doi:10.1038/nri3737

50. Schleimer RP, Schnaar RL, Bochner BS. Regulation of airway inflammation by siglec- 8 and siglec-9 sialoglycan ligand expression. Curr Opin Allergy Clin Immunol. 2016;16(1):24-30. doi:10.1097/ACI.0000000000000234

51. Zakeri A, Russo M. Dual role of toll-like receptors in human and experimental asthma models. Front Immunol. 2018;9:1027. doi:10.3389/fimmu.2018.01027

52. Pooe OJ, Kollisch G, Heine H, Shonhai A. Plasmodium falciparum heat shock protein 70 lacks immune modulatory activity. Protein Pept Lett. 2017;24(6):503-510. doi:10.2174/ 0929866524666170214141909

53. Ferat-Osorio E, Sanchez-Anaya A, Gutierrez-Mendoza M, et al. Heat shock protein 70 down-regulates the production of toll-like receptor-induced pro-inflammatory cytokines by a heat shock factor-1/constitutive heat shock element-binding factor-dependent mechanism. $J$ Inflamm (Lond). 2014;11(1):19. doi:10.1186/1476-9255-11-19

54. Bauer AK, Rondini EA, Hummel KA, et al. Identification of candidate genes downstream of TLR4 signaling after ozone exposure in mice: a role for heat-shock protein 70. Environ Health Perspect. 2011;119(8):1091-1097. doi:10.1289/ehp.1003326

55. Gorska MM. Natural killer cells in asthma. Curr Opin Allergy Clin Immunol. 2017;17(1):50-54. doi:10.1097/ACI.000000000 0000327

56. Farhadi N, Lambert L, Triulzi C, Openshaw PJM, Guerra N, Culley FJ. Natural killer cell NKG2D and granzyme B are critical for allergic pulmonary inflammation. J Allergy Clin Immunol. 2014;133(3):827-835.e3. doi:10.1016/j.jaci.2013.09.048

57. Ray A, Kolls JK. Neutrophilic inflammation in asthma and association with disease severity. Trends Immunol. 2017;38 (12):942-954. doi:10.1016/j.it.2017.07.003
58. Lommatzsch M, Julius $\mathrm{P}$, Kuepper $\mathrm{M}$, et al. The course of allergen-induced leukocyte infiltration in human and experimental asthma. J Allergy Clin Immunol. 2006;118(1):91-97. doi:10.1016/ j.jaci.2006.02.034

59. Lutfi R, Lewkowich IP, Zhou P, Ledford JR, Page K. The role of protease-activated receptor- 2 on pulmonary neutrophils in the innate immune response to cockroach allergen. J Inflamm (Lond). 2012;9(1):32. doi:10.1186/1476-9255-9-32

60. Patel DF, Piero T, Bruno N, et al. Neutrophil restrain allergic airway inflammation by limiting ILC2 function and monocyte-dendritic cell antigen presentation. Sci Immunol. 2019;4(41):eaax7006. doi:10.1126/sciimmunol.aax7006

61. Ortega E, Hinchado MD, Martin-Cordero L, Asea A. The effect of stress-inducible extracellular Hsp72 on human neutrophil chemotaxis: a role during acute intense exercise. Stress. 2009;12 (3):240-249. doi:10.1080/10253890802309853

62. Assimon VA, Gillies AT, Rauch JN, Gestwicki JE. Hsp70 protein complexes as drug targets. Curr Pharm Des. 2013;19(3):404-417. doi:10.2174/138161213804143699

63. Fan GH, Yang W, Sai J, Richmond A. Hsc/Hsp70 interacting protein (hip) associates with CXCR2 and regulates the receptor signaling and trafficking. $J$ Biol Chem. 2002;277(8):6590-6597. doi:10.1074/jbc.M110588200

64. Ciepiela O, Ostafin M, Demkow U. Neutrophils in asthma-a review. Respir Physiol Neurobiol. 2015;209:13-16. doi:10.1016/ j.resp.2014.12.004

65. Dworski R, Simon HU, Hoskins A, Yousefi S. Eosinophil and neutrophil extracellular DNA traps in human allergic asthmatic airways. J Allergy Clin Immunol. 2011;127(5):1260-1266. doi:10.1016/j.jaci.2010.12.1103

66. Fox S, Leitch AE, Duffin R, Haslett C, Rossi AG. Neutrophil apoptosis: relevance to the innate immune response and inflammatory disease. J Innate Immun. 2010;2(3):216-227. doi:10.1159/ 000284367

67. Troyanova NI, Shevchenko MA, Boyko AA, et al. Modulating effect of extracellular HSP70 on generation of reactive oxigen species in populations of phagocytes. Russ J Bioorg Chem. 2015;41(3):271-279. doi:10.1134/S1068162015030097

68. Yurinskaya MM, Evgen'ev MB, Antonova OY, Vinokurov MG. Exogenous heat shock protein HSP70 suppresses bacterial pathogen-induced activation of human neutrophils. Dokl Biochem Biophys. 2010;435:316-319. doi:10.1134/S16076729 10060098

69. Sofoluwe A, Bacchetta M, Badaoui M, Kwak BR, Chanson M. ATP amplifies NADPH-dependent and -independent neutrophil extracellular trap formation. Sci Rep. 2019;9(1):16556. doi:10.1038/s41598-019-53058-9

70. Woytschak J, Keller N, Krieg C, et al. Type 2 interleukin-4 receptor signaling in neutrophils antagonizes their expansion and migration during infection and inflammation. Immunity. 2016;45(1):172-184. doi:10.1016/j.immuni.2016.06.025

71. Li Y, Shi XL, Cheng ZY, Li GP, Zhong S, Chen Z. HSP70/CD80 DNA vaccine inhibits airway remodeling by regulating the transcription factors T-bet and GATA-3 in a murine model of chronic asthma. Arch Med Sci. 2013;9(5):906-915. doi:10.5114/ aoms.2013.33180

72. Roche PA, Furuta K. The ins and outs of MHC class II-mediated antigen processing and presentation. Nat Rev Immunol. 2015;15 (4):203-216. doi:10.1038/nri3818

73. Afshar R, Medoff BD, Luster AD. Allergic asthma: a tale of many T cells. Clin Exp Allergy. 2008;38(12):1847-1857. doi:10.1111/j.1365-2222.2008.03119.x

74. Lambrecht BN. Dendritic cells and the regulation of the allergic immune response. Allergy. 2005;60(3):271-282. doi:10.1111/ j.1398-9995.2005.00708.x 
75. Srivastava P. Roles of heat-shock proteins in innate and adaptive immunity. Nat Rev Immunol. 2002;2(3):185-194. doi:10.1038/ nri749

76. Multhoff G. Heat shock protein 70 (Hsp70): membrane location, export and immunological relevance. Methods. 2007;43 (3):229-237. doi:10.1016/j.ymeth.2007.06.006

77. Shevtsov M, Multhoff G. Heat shock protein-peptide and HSP-based immunotherapies for the treatment of cancer. Front Immunol. 2016;7:171. doi:10.3389/fimmu.2016.00171

78. Motta A, Schmitz C, Rodrigues L, et al. Mycobacterium tuberculosis heat-shock protein 70 impairs maturation of dendritic cells from bone marrow precursors, induces interleukin-10 production and inhibits T-cell proliferation in vitro. Immunology. 2007;121 (4):462-472. doi:10.1111/j.1365-2567.2007.02564.x

79. Rohrer KM, Haug M, Schworer D, Kalbacher H, Holzer U. Mutations in the substrate binding site of human heat-shock protein 70 indicate specific interaction with HLA-DR outside the peptide binding groove. Immunology. 2014;142(2):237-247. doi:10.1111/imm.12249

80. Stocki P, Morris NJ, Preisinger C, et al. Identification of potential HLA class I and class II epitope precursors associated with heat shock protein 70 (HSPA). Cell Stress Chaperones. 2010;15 (5):729-741. doi:10.1007/s12192-010-0184-Z

81. Berings M, Karaaslan C, Altunbulakli C, et al. Advances and highlights in allergen immunotherapy: on the way to sustained clinical and immunologic tolerance. J Allergy Clin Immunol. 2017;140(5):1250-1267. doi:10.1016/j.jaci.2017.08.025

82. Wambre E, DeLong JH, James EA, et al. Specific immunotherapy modifies allergen-specific $\mathrm{CD} 4(+)$ T-cell responses in an epitope-dependent manner. J Allergy Clin Immunol. 2014;133 (3):872-879.e7. doi:10.1016/j.jaci.2013.10.054

83. Brenu EW, Staines DR, Tajouri L, Huth T, Ashton KJ, MarshallGradisnik SM. Heat shock proteins and regulatory $\mathrm{T}$ cells. Autoimmune Dis. 2013;2013:813256. doi:10.1155/2013/813256

84. Wieten L, van der Zee R, Spiering R, et al. A novel heat-shock protein coinducer boosts stress protein Hsp70 to activate $\mathrm{T}$ cell regulation of inflammation in autoimmune arthritis. Arthritis Rheum. 2010;62(4):1026-1035. doi:10.1002/art.27344

85. Mantel PY, Kuipers H, Boyman O, et al. GATA3-driven Th2 responses inhibit TGF-beta1-induced FOXP3 expression and the formation of regulatory T cells. PLoS Biol. 2007;5(12):e329. doi:10.1371/journal.pbio.0050329

86. Joly AL, Wettstein G, Mignot G, Ghiringhelli F, Garrido C. Dual role of heat shock proteins as regulators of apoptosis and innate immunity. J Innate Immun. 2010;2(3):238-247. doi:10.1159/ 000296508

87. Kampinga HH, Craig EA. The HSP70 chaperone machinery: $\mathrm{J}$ proteins as drivers of functional specificity. Nat Rev Mol Cell Biol. 2010;11(8):579-592. doi:10.1038/nrm2941

88. Rosenzweig R, Nillegoda NB, Mayer MP, Bukau B. The Hsp70 chaperone network. Nat Rev Mol Cell Biol. 2019;20(11):665-680. doi:10.1038/s41580-019-0133-3

89. Terrab L, Wipf P. Hsp70 and the unfolded protein response as a challenging drug target and an inspiration for probe molecule development. ACS Med Chem Lett. 2020;11(3):232-236. doi:10.1021/acsmedchemlett.9b00583

90. Osorio F, Lambrecht B, Janssens S. The UPR and lung disease. Semin Immunopathol. 2013;35:293-306. doi:10.1007/s00281013-0368-6

91. Miao K, Zhang L, Pan T, Wang Y. Update on the role of endoplasmic reticulum stress in asthma. Am J Transl Res. 2020;12 (4):1168-1183.

92. Kim SR, Kim DI, Kang MR, et al. Endoplasmic reticulum stress influences bronchial asthma pathogenesis by modulating nuclear factor $\kappa \mathrm{B}$ activation. $J$ Allergy Clin Immunol. 2013;132 (6):1397-1408. doi:10.1016/j.jaci.2013.08.041
93. Zeki AA, Yeganeh B, Kenyon NJ, Post M, Ghavami S Autophagy in airway diseases: a new frontier in human asthma? Allergy. 2016;71(1):5-14. doi:10.1111/all.12761

94. Fernández-Fernández MR, Gragera M, Ochoa-Ibarrola L, Quintana-Gallardo L, Valpuesta JM. Hsp70 - a master regulator in protein degradation. FEBS Lett. 2017;591(17):2648-2660. doi:10.1002/1873-3468.12751

95. Lukaszewicz A, Niechoda A, Zarzecki M, Cwiklinska M, Holownia A. Co-expression of Hsp70 protein and autophagy marker protein LC3 in A549 cells and THP1 cells exposed to nanoparticles of air pollution. Adv Exp Med Biol. 2020;1271:61-68.

96. Ornatowski W, Lu Q, Yegambaram M, et al. Complex interplay between autophagy and oxidative stress in the development of pulmonary disease. Redox Biol. 2020;36:101679. doi:10.1016/j. redox.2020.101679

97. McAlinden KD, Deshpande DA, Ghavami S, et al. Autophagy activation in asthma airways remodeling. Am J Respir Cell Mol Biol. 2019;60(5):541-553. doi:10.1165/rcmb.2018-0169OC

98. Liu JN, Suh DH, Trinh HK, Chwae YJ, Park HS, Shin YS. The role of autophagy in allergic inflammation: a new target for severe asthma. Exp Mol Med. 2016;48(7):e243. doi:10.1038/ emm.2016.38

99. Menoret A. Purification of recombinant and endogenous HSP70s. Methods. 2004;32(1):7-12. doi:10.1016/S1046-2023(03)00180-4

100. Hurley JH. The sugar kinase/heat shock protein $70 /$ actin super family: implications of conserved structure for mechanism. Annu Rev Biophys Biomol Struct. 1996;25:137-162. doi:10.1146/ annurev.bb.25.060196.001033

101. Vogel M, Bukau B, Mayer MP. Allosteric regulation of Hsp70 chaperones by a proline switch. Mol Cell. 2006;21(3):359-367. doi:10.1016/j.molcel.2005.12.017

102. Theyssen H, Schuster HP, Packschies L, Bukau B, Reinstein J. The second step of ATP binding to DnaK induces peptide release. J Mol Biol. 1996;263(5):657-670. doi:10.1006/jmbi.1996.0606

103. Kityk R, Kopp J, Sinning I, Mayer MP. Structure and dynamics of the ATP-bound open conformation of Hsp70 chaperones. Mol Cell. 2012;48(6):863-874. doi:10.1016/j.molcel.2012.09.023

104. Kepp O, Loos F, Liu P, Kroemer G. Extracellular nucleosides and nucleotides as immunomodulators. Immunol Rev. 2017;280 (1):83-92. doi:10.1111/imr.12571

105. Willart MA, Lambrecht BN. The danger within: endogenous danger signals, atopy and asthma. Clin Exp Allergy. 2009;39 (1):12-19. doi:10.1111/j.1365-2222.2008.03118.x

106. Idzko M, Hammad H, van Nimwegen M, et al. Extracellular ATP triggers and maintains asthmatic airway inflammation by activating dendritic cells. Nat Med. 2007;13(8):913-919. doi:10.1038/nm1617

107. Gorini S, Gatta L, Pontecorvo L, Vitiello L, la Sala A. Regulation of innate immunity by extracellular nucleotides. Am J Blood Res. 2013;3(1):14-28

108. Yegutkin GG. Nucleotide- and nucleoside-converting ectoenzymes: important modulators of purinergic signalling cascade. Biochim Biophys Acta. 2008;1783(5):673-694. doi:10.1016/j. bbamcr.2008.01.024

109. Zimmermann H, Zebisch M, Strater N. Cellular function and molecular structure of ecto-nucleotidases. Purinergic Signal. 2012;8(3):437-502.

110. Vitiello L, Gorini S, Rosano G, la Sala A. Immunoregulation through extracellular nucleotides. Blood. 2012;120(3):511-518. doi:10.1182/blood-2012-01-406496

111. Bolkhovitina EL, Sapozhnikov AM, Shevchenko MA. Effect of heat shock protein 70 on ATP-induced dendritic cell chemotaxis. Russ J Immunol. 2013;7((16)(2-3)):342-343.

112. Goloudina AR, Demidov ON, Garrido C. Inhibition of HSP70: a challenging anti-cancer strategy. Cancer Lett. 2012;325 (2):117-124. doi:10.1016/j.canlet.2012.06.003 
113. Breitenbach M, Simon-Nobbe B. The allergens of Cladosporium herbarum and Alternaria alternata. Chem Immunol. 2002;81:48-72.

114. Shen HD, Au LC, Lin WL, Liaw SF, Tsai JJ, Han SH. Molecular cloning and expression of a Penicillium citrinum allergen with sequence homology and antigenic crossreactivity to a hsp 70 human heat shock protein. Clin Exp Allergy. 1997;27 (6):682-690. doi:10.1111/j.1365-2222.1997.tb01197.x

115. Aki T, Fujikawa A, Wada T, et al. Cloning and expression of cDNA coding for a new allergen from the house dust mite, dermatophagoides farinae: homology with human heat shock cognate proteins in the heat shock protein 70 family. J Biochem. 1994;115(3):435-440. doi:10.1093/oxfordjournals.jbchem.a124356

116. An S, Chen LL, Long CB, et al. Dermatophagoides farinae allergens diversity identification by proteomics. Mol Cell Proteomics. 2013;12(7):1818-1828. doi:10.1074/mcp.M112.027136

117. Liu XY, Yang KY, Wang MQ, et al. High-quality assembly of Dermatophagoides pteronyssinus genome and transcriptome reveals a wide range of novel allergens. $J$ Allergy Clin Immunol. 2018;141(6):2268-2271. doi:10.1016/j.jaci.2017.11.038
118. Cui Y, Yu L, Teng F, et al. Transcriptomic/proteomic identification of allergens in the mite Tyrophagus putrescentiae. Allergy. 2016;71(11):1635-1639. doi:10.1111/all.12999

119. Gruehn S, Suphioglu C, O'Hehir RE, Volkmann D. Molecular cloning and characterization of hazel pollen protein $(70 \mathrm{kD})$ as a luminal binding protein (BiP): a novel cross-reactive plant allergen. Int Arch Allergy Immunol. 2003;131(2):91-100. doi:10.1159/000070924

120. Zhang L, Muradia G, De Vouge MW, Rode H, Vijay HM. An allergenic polypeptide representing a variable region of hsp 70 cloned from a cDNA library of Cladosporium herbarum. Clin Exp Allergy. 1996;26(1):88-95. doi:10.1111/j.1365-2222.1996. tb00060.x

121. Simon-Nobbe B, Denk U, Poll V, Rid R, Breitenbach M. The spectrum of fungal allergy. Int Arch Allergy Immunol. 2008;145 (1):58-86. doi:10.1159/000107578

\section{Publish your work in this journal}

The Journal of Asthma and Allergy is an international, peer-reviewed open-access journal publishing original research, reports, editorials and commentaries on the following topics: Asthma; Pulmonary physiology; Asthma related clinical health; Clinical immunology and the immunological basis of disease; Pharmacological interventions and new therapies. The manuscript management system is completely online and includes a very quick and fair peer-review system, which is all easy to use. Visit http://www.dovepress.com/testimonials.php to read real quotes from published authors. 